\title{
Combination and QCD analysis of charm and beauty production cross section measurements in deep inelastic ep scattering at HERA
}

\author{
O. Zenaiev* on behalf of the $\mathrm{H} 1$ and ZEUS collaborations \\ DESY, Notkestr. 85, Hamburg 22607, Germany \\ E-mail: oleksandr.zenaiev@desy.de
}

\begin{abstract}
Measurements of open charm and beauty production cross sections in deep inelastic ep scattering at HERA from the H1 and ZEUS Collaborations are combined and reduced cross sections are obtained. The combination method accounts for the correlations of the statistical and systematic uncertainties among the different datasets. Perturbative QCD calculations are compared to the combined data. A next-to-leading order QCD analysis is performed using these data together with the combined inclusive deep inelastic scattering cross sections from HERA. The running charm- and beauty-quark masses are determined.
\end{abstract}

The 39th International Conference on High Energy Physics (ICHEP2018)

4-11 July, 2018

Seoul, Korea

${ }^{*}$ Speaker. 
Measurements of open charm and beauty production in deep inelastic electron-proton scattering (DIS) at HERA provide important input for stringent tests of QCD. This analysis [1] is an extension of the previous H1 and ZEUS combination [2] of charm measurements in DIS [3, 4, 5, 6, $7,8,9,10]$ with new charm and beauty data $[11,12,13,14,15,3]$. The reduced charm, $\sigma_{\text {red }}^{c \bar{c}}$, and beauty, $\sigma_{\text {red }}^{b \bar{b}}$, cross sections are combined to create one consistent set of charm and beauty cross sections in the kinematic range of photon virtuality $2.5 \leq Q^{2} \leq 2000 \mathrm{GeV}^{2}$ and Bjorken scaling variable $3 \times 10^{-5} \leq x_{\mathrm{Bj}} \leq 5 \times 10^{-2}$ :

$$
\sigma_{\mathrm{red}}^{Q \bar{Q}}=\frac{\mathrm{d}^{2} \sigma^{Q \bar{Q}}}{\mathrm{~d} x_{\mathrm{Bj}} \mathrm{d} Q^{2}} \cdot \frac{x_{\mathrm{Bj}} Q^{4}}{2 \pi \alpha^{2}\left(1+(1-y)^{2}\right)} .
$$

Here $Q \bar{Q}$ stands for $c \bar{c}$ or $b \bar{b}$ quark-antiquark pairs, and $y$ is the inelasticity. The reduced cross sections are determined at common $\left(x_{\mathrm{Bj}}, Q^{2}\right)$ points from the visible cross sections, defined as the $D-, \mu-, e$ - or jet-production cross sections in a particular kinematic range, using theoretical predictions obtained with the HVQDIS programme [17] and extended with fragmentation models to provide hadron level cross sections as described elsewhere $[2,18]$. The updated fragmentation fractions of $c$ quarks into specific charmed hadrons are used [19] and the branching fractions of semi-leptonic decays of heavy-quarks to a muon or electron are taken from the PDG [20]. A total $\chi^{2}$ of 149 for 187 degrees of freedom (dof) is obtained in the combination indicating consistency of input data and conservative estimates of the uncertainties. The individual datasets as well as the results of the combination are shown in Fig. 1.
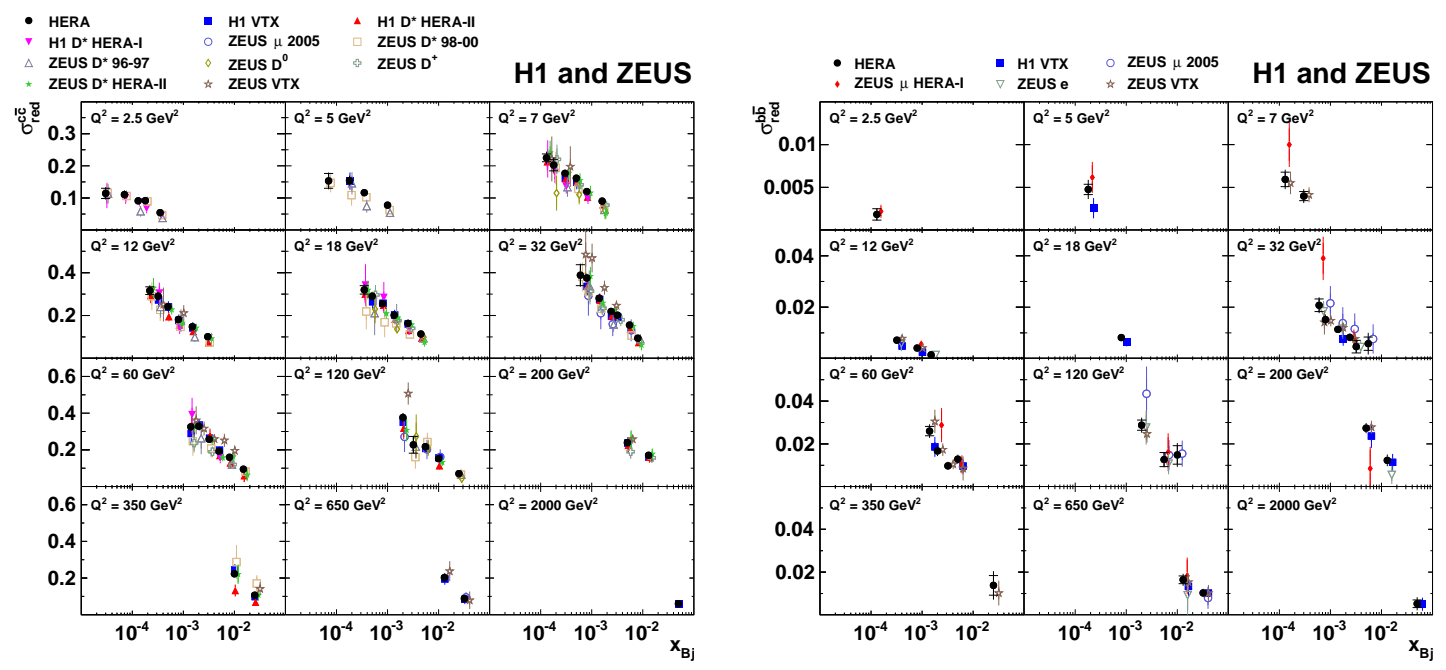

Figure 1: Combined reduced charm (left) and beauty (right) cross sections (full circles) as a function of $x_{\mathrm{Bj}}$ for different values of $Q^{2}$. The inner error bars indicate the uncorrelated part of the uncertainties and the outer error bars represent the total uncertainties. The input measurements are also shown by the different markers. For presentation purposes each individual measurement is shifted in $x_{\mathrm{Bj}}$.

The combined beauty and charm data are included in a QCD analysis at NLO, performed using XFITTER [21], together with the combined HERA inclusive DIS data [16]. The theoretical predictions in the fixed-flavour-number scheme (FFNS) for the HERA data are obtained using the 

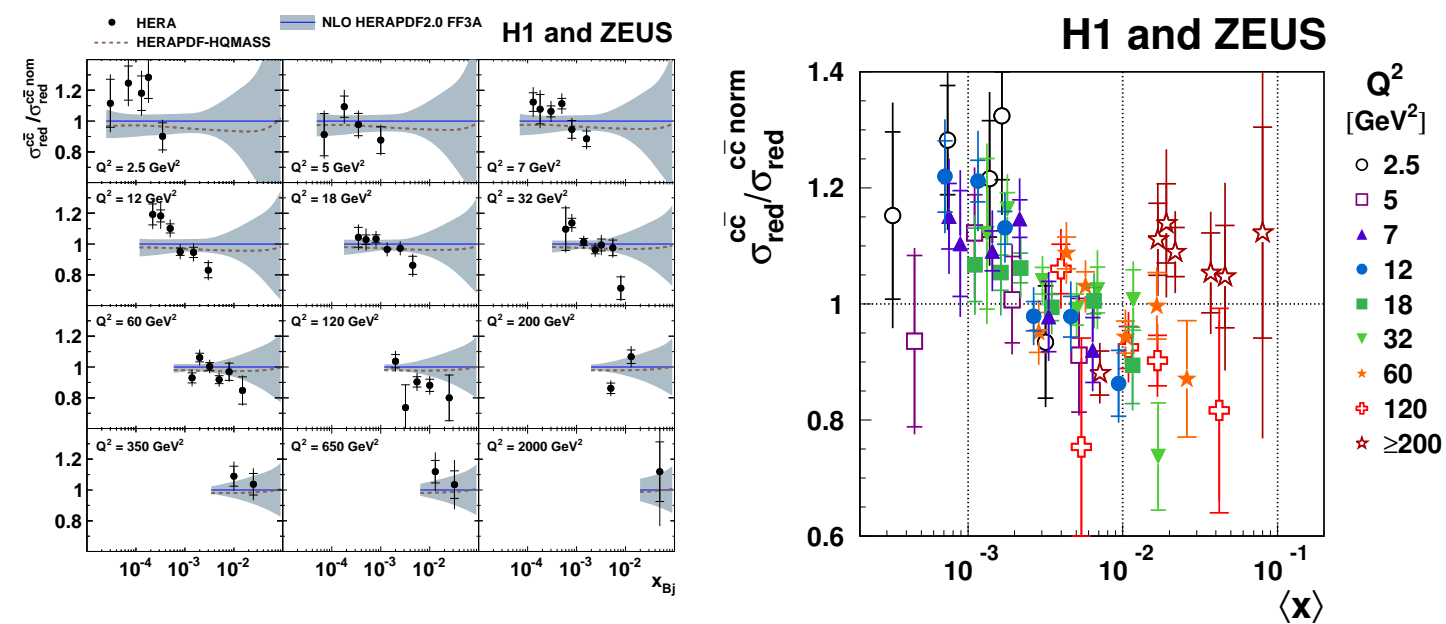

Figure 2: (left) Combined reduced charm cross sections (full circles) as a function of $x_{\mathrm{Bj}}$ for given values of $Q^{2}$, compared to the NLO QCD FFNS predictions based on HERAPDF-HQMASS (dashed lines) and on HERAPDF2.0 FF3A (solid lines). The shaded bands on the HERAPDF2.0 FF3A predictions show the theory uncertainties obtained by adding PDF, scale and charm-quark mass uncertainties in quadrature. (right) Ratio of the combined reduced charm cross sections to the respective NLO FFNS cross-section predictions as a function of the partonic $\langle x\rangle$ for different values of $Q^{2}$.

OPENQCDRAD programme [22] interfaced in the XFITTER framework. The number of active flavours is set to $n_{f}=3$ at all scales. The heavy-quark masses are left free in the fit. For the heavy-flavour contributions to the inclusive DIS cross sections the pQCD scales are set to $\mu_{r}=$ $\mu_{f}=\sqrt{Q^{2}+4 m_{\mathrm{Q}}^{2}}$, and for the light-flavour contributions the scales are set to $\mu_{r}=\mu_{f}=Q$. The strong coupling is set to $\alpha_{s}^{n_{f}=3}\left(M_{Z}\right)=0.106$, corresponding to $\alpha_{s}^{n_{f}=5}\left(M_{Z}\right)=0.118$. The $Q^{2}$ range of the inclusive HERA data is restricted to $Q^{2}>Q_{\min }^{2}=3.5 \mathrm{GeV}^{2}$. The $\chi^{2}$ definition and PDF parameterisation follow the HERAPDF2.0 analysis [16]. The PDF uncertainties are estimated as in the general approach of HERAPDF2.0 [16] in which the experimental, model, and parametrisation uncertainties are taken into account.

The extracted heavy-quark masses are:

$$
\begin{aligned}
& \left.m_{c}\left(m_{c}\right)=1.290_{-0.041}^{+0.046}(\exp / \mathrm{fit})_{-0.014}^{+0.062}(\text { model })_{-0.031}^{+0.003} \text { (parameterisation }\right) \mathrm{GeV} \\
& \left.m_{b}\left(m_{b}\right)=4.049_{-0.109}^{+0.104}(\exp / \mathrm{fit})_{-0.032}^{+0.090}(\text { model })_{-0.031}^{+0.001} \text { (parameterisation }\right) \mathrm{GeV}
\end{aligned}
$$

The model uncertainties are dominated by theoretical uncertainties arising from the scale variations. The fit yields $\chi^{2} /$ dof $=1435 / 1208$. The resulting fit is termed HERAPDF-HQMASS. The resulting theoretical predictions for the charm data are shown in Fig. 2 (left). The FFNS calculations reasonably describe the charm data, although in the kinematic range where the data are very precise the data show a $x_{\mathrm{Bj}}$ dependence somewhat steeper than predicted by the calculations. The same trend was observed for predictions using different PDF sets or different QCD calculations at NLO or NNLO [1]. Predictions obtained using the variable-flavour-number scheme were also compared to the data; overall, the description is not improved with respect to the FFNS reference calculations [1]. In Fig. 2 (right), the ratio of the measured reduced cross sections to the theoretical predictions is shown as a function of $\langle x\rangle$ instead of $x_{\mathrm{Bj}}$, where $\langle x\rangle$ is the geometric mean of the 
folding variable $x$ calculated at NLO with HVQDIS. A deviation from the reference calculation is evident, showing a steeper slope in $\langle x\rangle$ in the range $0.0005 \lesssim\langle x\rangle \lesssim 0.01$, consistent with being independent of $Q^{2}$. Due to the larger experimental uncertainties, no conclusion can be drawn for the beauty data [1].

In summary, measurements of beauty and charm production cross sections in deep inelastic ep scattering by the $\mathrm{H} 1$ and ZEUS experiments were combined at the level of reduced cross sections, accounting for their statistical and systematic correlations. The beauty cross sections have been combined for the first time. The data sets were found to be consistent and the combined data have significantly reduced uncertainties. The combined data were compared to perturbative QCD predictions, which are found to describe the data reasonably well. The running charm and beauty masses were extracted in the QCD analysis using the inclusive and new combined charm and beauty HERA data.

\section{References}

[1] H. Abramowicz et al. [H1 and ZEUS Coll.], Eur. Phys. J. C78, 473 (2018) [arXiv:1804.01019]

[2] F. D. Aaron et al. [H1 and ZEUS Coll.], Eur. Phys. J. C73, 2311 (2013) [arXiv:1211.1182].

[3] F. D. Aaron et al. [H1 Coll.], Eur. Phys. J. C65, 89 (2010) [arXiv:0907.2643].

[4] A. Aktas et al. [H1 Coll.], Eur. Phys. J. C51, 271 (2007) [hep-ex/0701023].

[5] F. D. Aaron et al. [H1 Coll.], Eur. Phys. J. C71, 1769 (2011) [arXiv:1106.1028].

[6] F. D. Aaron et al. [H1 Coll.], Phys. Lett. B686, 91 (2010) [arXiv:0911.3989].

[7] J. Breitweg et al. [ZEUS Coll.], Eur. Phys. J. C12, 35 (2000) [hep-ex/9908012].

[8] S. Chekanov et al. [ZEUS Coll.], Phys. Rev. D69, 012004 (2004) [hep-ex/0308068].

[9] S. Chekanov et al. [ZEUS Coll.], Eur. Phys. J. C63, 171 (2009) [arXiv:0812.3775].

[10] S. Chekanov et al. [ZEUS Coll.], Eur. Phys. J. C65, 65 (2010) [arXiv:0904.3487].

[11] H. Abramowicz et al. [ZEUS Coll.], JHEP 05, 023 (2013) [arXiv:1302.5058].

[12] H. Abramowicz et al. [ZEUS Coll.], JHEP 05, 097 (2013) [arXiv:1303.6578].

[13] H. Abramowicz et al. [ZEUS Coll.], JHEP 09, 127 (2014) [arXiv:1405.6915].

[14] H. Abramowicz et al. [ZEUS Coll.], Eur. Phys. J. C71, 1573 (2011) [arXiv:1101.3692].

[15] H. Abramowicz et al. [ZEUS Coll.], Eur. Phys. J. C69, 347 (2010) [arXiv:1005.3396].

[16] H. Abramowicz et al. [H1 and ZEUS Coll.], Eur. Phys. J. C75, 580 (2015) [arXiv:1506.06042].

[17] B. W. Harris and J. Smith, Phys. Rev. D57, 2806 (1998) [hep-ph/9706334].

[18] P. Nason and C. Oleari, Nucl. Phys. B565, 245 (2000) [hep-ph/9903541].

[19] M. Lisovyi et al., Eur. Phys. J. C76, 397 (2016) [arXiv:1509.01061].

[20] C. Patrignani et al. [Particle Data Group], Chin. Phys. C40, 100001 (2016).

[21] S. Alekhin et al., Eur. Phys. J. C75, 304 (2015) [arXiv:1410.4412], www . xfitter.org (v2.0.0).

[22] S. Alekhin, J. Blümlein, S. Moch, Phys. Rev. D86, 054009 (2012) [arXiv:1202.2281]; I. Bierenbaum, J. Blümlein, S. Klein, Phys. Lett. B672, 401 (2009) [arXiv:0901.0669]; S. Alekhin, OPENQCDRAD-1.5, http://www-zeuthen. desy. de/ alekhin/OPENQCDRAD (v2.1). 